\title{
哥iresalce
}

\section{A experiência com o uso do e-learning na aprendizagem baseada em problemas de um curso de medicina}

\author{
The experience with the use of e-learning in problem-based learning in an undergraduate \\ medical school
Nara Miranda Portela ${ }^{1}$, Juliana Martins Barbosa da Silva Costa ${ }^{2}$, Gustavo Sérgio de Godoy Magalhães ${ }^{3}$

\section{Resumo}

Introdução: A Aprendizagem Baseada em Problema (ABP) vem estimulando transformações nos currículos de escolas médicas desde a década de 1960. Nos dias atuais, novas tecnologias educacionais (TE) se multiplicam e potencializam diferentes modos de ensinar e aprender. Nos cursos de saúde, o avanço das TE produziu a ABP Híbrida, metodologia que usa recursos do e-learning, combinando atividades a distância e presenciais, para promover o ensino-aprendizagem de forma ativa. Objetivo: Relatar a experiência do uso de recursos de e-learning na ABP em um curso de medicina. Método: Descrever e analisar a experiência do uso do e-learning incorporado como estratégia facilitadora e integradora da $A B P$ no curso de graduação em medicina recém-implantado no interior no Nordeste brasileiro. Resultado: Estratégias de e-learning são empregadas em todos os passos da $A B P$, seja como ferramenta de registro, seja como mediação de discussões virtuais, facilitando a interação e construção coletiva do conhecimento, oportunizando, assim, experiências diversas de aprendizado para estudantes com diferentes estilos de aprendizagem. Conclusão: A flexibilidade de tempo e espaço, ao realizar atividades à distância, amplia as possibilidades do ensinar e aprender, tocando aspectos como interação entre os estudantes, profundidade de discussões e envolvimento no processo tutorial.

Palavras-Chave: Aprendizagem Baseada em Problemas. Educação a distância. Educação Médica.

\begin{abstract}
Introduction: The Problem-Based Learning (PBL) has been stimulating transformations in medical school curricula since the 1960s. Nowadays, new educational technologies (ET) multiply and enhance different ways of teaching and learning. In health courses, the advancement of ET produced the Hybrid PBL, a methodology that uses e-learning resources combining distance and classroom activities to actively promote teaching-learning. Objective: To report the experience of using e-learning resources in PBL in a medical school. Method: Describe and analyze the experience of using embedded e-learning as a facilitating and integrating strategy for PBL in the undergraduate medical course recently

1 Doutora 1. Núcleo de Ciências da Vida do Campus do Agreste, UFPE. Email: nara.portela@ufpe.br 2 Doutora 2. Núcleo de Ciências da Vida do Campus do Agreste, UFPE. Email: julimartins.costa@gmail.com 3 Mestre 3. Núcleo de Ciências da Vida do Campus do Agreste, UFPE. Email: gustavo.magalhaes@ufpe.br Correspondência: Núcleo de Ciência da Vida, Campus Agreste da Universidade Federal de Pernambuco. Rodovia BR 104, km 62, S/N, Nova Caruaru, Caruaru, PE - CEP 55014-908.
\end{abstract}


implemented in the Northeast of Brazil. Result: E-learning strategies are used in all steps of $P B L$, either as a registration tool or as a mediation for virtual discussions, facilitating the interaction and collective construction of knowledge, providing different learning experiences for students with different learning styles. Conclusion: The flexibility of time and space when carrying out activities at a distance expands the possibilities of teaching and learning by touching aspects such as interaction between students, depth of discussions and involvement in the tutorial process.

Keywords: Problem-Based Learning. Education, Distance. Education, Medical.

\section{Introdução}

A Aprendizagem Baseada em Problema (ABP), do inglês Problem Based Learning, é uma estratégia de ensinoaprendizagem centrada no estudante, introduzida no ensino de Ciências da Saúde na Universidade de McMaster, Canadá, na década de $1960^{1}$. Na ABP, pequenos grupos de estudantes são apresentados a problemas que precisam ser solucionados, colaborativamente, por meio de uma sequência de passos bem definida.

A ABP estimula 0 estudante a desenvolver habilidades para gerenciar o próprio aprendizado, buscar ativamente as informações, integrar o conhecimento, identificar e explorar novas áreas de conhecimento. Assim, o estudante desenvolve, não só as competências necessárias para a prática profissional, mas também, as necessárias para aprender ao longo da vida ${ }^{1,2}$.

O processo da ABP ocorre em ciclos divididos em três momentos ${ }^{2}$. Na primeira sessão tutorial, os estudantes definem os objetivos de aprendizagem a partir da análise do problema. Em seguida, os membros do grupo partem para o estudo individual aprofundado. O ciclo tutorial da ABP é finalizado em uma segunda sessão tutorial, na qual os novos conhecimentos dos estudantes são usados na resolução dos problemas levantados no primeiro encontro. Desde a apresentação da ABP à comunidade científica, há uma convergência da literatura em relação aos três momentos do ciclo tutorial; no entanto, não há um consenso no número de passos que compõem cada um destes momentos, nem na atividade realizada em cada passo $^{3,4,5,6,7}$.

Como se trata de um modelo que não é fixo nem fechado, a ABP pode ser adaptada às condições específicas de cada currículo e ao surgimento de novas tecnologias educacionais, como é o caso do Problem-based e-learning (ePBL) ${ }^{8}$. Em um sentido mais amplo, e-learning é o uso da internet na educação. No entanto, ele vai além da simples disponibilização online de documentos digitais para os alunos. $\mathrm{O}$ e-learning engloba uma abordagem pedagógica que, tipicamente, busca ser flexível, engajadora, centrada no aluno, assíncrona (na maioria das vezes), que incentiva a interação, colaboração e 
comunicação a distância entre os atores envolvidos no aprendizado (professoraluno, aluno-aluno e professor-professor) ${ }^{8}$. $\mathrm{O}$ ePBL, então, se trata da ABP que usa recursos do e-learning para promover 0 ensino-aprendizagem, sendo o processo completamente online ou uma combinação de atividades a distância e presenciais em diferentes proporções, como é o caso da aprendizagem híbrida (do inglês, blended learning $)^{9}$, apresentada neste artigo como ABP híbrida.

A partir do objetivo pedagógico, os recursos do e-learning podem se limitar à disponibilização de conteúdo online (material didático em texto, áudio, vídeo e links para páginas externas, biblioteca virtual, banco de dados e instruções de atividades) ou envolver processos tais como: (1) Comunicação e Interação promovidas pelo uso dos chats e fóruns de discussão; (2) Criação Individual de Conteúdo nos blogs e nas ferramentas de portfólio; (3) Criação Colaborativa de Conteúdo nas ferramentas wiki; e a (4) Avaliação de Aprendizagem através de tarefas, lições interativas, quiz, simulações, testes e questionários online $e^{8,9}$.

O e-learning pode acontecer em diversas plataformas, por exemplo: uma atividade baseada em chat pode ser realizada usando um aplicativo de troca instantânea de mensagens pelo celular; uma discussão no formato de fórum pode acontecer em uma rede social; vídeo-aulas podem ser disponibilizadas em um site de compartilhamento de vídeos e provas online podem ser construídas usando serviços de formulários eletrônicos ${ }^{8,9}$ No entanto, quando se tem um currículo integrado em uma modalidade híbrida, gerenciar e integrar diferentes plataformas se torna um desafio. Nesse contexto, é comum que as instituições de ensino optem por ter um Ambiente Virtual de Aprendizagem (AVA), um conjunto integrado de ferramentas e serviços que ampliam as possibilidades do ensinar e do aprender ao favorecer a realização de múltiplas atividades online 8,11 .

A implantação de novas tecnologias geralmente introduz tensões e com $e$ learning não é diferente. Alguns currículos podem integrá-lo apenas para realizar atividades pré-existentes de maneira mais eficiente ou mais rápida. Outros buscam formas disruptivas de pensar e trabalhar, proporcionadas pelo uso de novas tecnologias educacionais ${ }^{8}$. Em ambos os cenários, a educação, e não a tecnologia é o objetivo principal; no entanto, nem sempre é possível prever os resultados de uma mudança de metodologia de ensino.

Atualmente (2019) existem no Brasil 337 Escolas Médicas, 80 (23,7\%) das quais declaram utilizar o método $A B P$, como base do currículo ou de forma combinada com o ensino tradicional ${ }^{12}$. Apesar da tendência atual de valorização do desenvolvimento gradual de competências profissionais em situações da vida real - que demandem conhecimento, habilidades e atitudes - o 
ABP continua sendo usado em grau maior ou menor, em muitas escolas ao redor do mundo ${ }^{13}$.

Este trabalho tem por objetivo relatar a experiência do uso de recursos de $e$ learning na ABP em um curso de medicina recém-implantado no interior do Nordeste do Brasil.

\section{Métodos}

Trata-se de um relato de experiência do uso de recursos do e-learning incorporados como estratégia facilitadora e integradora da ABP, desenvolvida no curso de graduação em medicina do campus Agreste da Universidade Federal de Pernambuco, sediado no município de Caruaru, maior cidade do interior do estado. A experiência observada e analisada se deu no período entre março de 2014 e dezembro de 2019.

O curso de medicina faz parte do Núcleo de Ciências da Vida do Centro Acadêmico do Agreste e foi implantado em 2014. Apresenta única entrada com 80 estudantes e está dividido em dois ciclos. O primeiro com duração de quatro anos, composto por módulos temáticos e prática de integração ensino-serviço na comunidade. O segundo ciclo compreende dois anos de internato que ocorre na rede de serviços de saúde municipal e estadual. A ABP é utilizada como estratégia pedagógica no primeiro ciclo do curso, isto é, do primeiro ao oitavo semestre. Cerca de 40 tutores e 320 estudantes/ano utilizam essa metodologia.

O relato foi estruturado em três etapas. A primeira trata de uma breve descrição do curso e do seu desenvolvimento curricular. A segunda, da apresentação da ABP, sua organização, sequência de passos e a inserção do elearning como dinamizador deste processo. Por fim, na terceira e última etapa, realizou-se uma reflexão crítica sobre os fatores facilitadores e limites da utilização de ferramentas do e-learning como parte integrante da $\mathrm{ABP}$, a partir da vivência docente.

\section{Resultados}

O curso de graduação em Medicina do Núcleo de Ciências da Vida, do Campus do Agreste da Universidade Federal de Pernambuco, iniciou suas ações, em 2014, em um contexto de interiorização do ensino superior, com o intuito de responder às Diretrizes Curriculares Nacionais ${ }^{14}$. Apresenta um Projeto Pedagógico que, entre outros aspectos, fundamenta-se na aprendizagem ativa ${ }^{15}$. Nessa perspectiva, tal projeto busca o ensino centrado nas necessidades de aprendizagem dos estudantes, mediado por situaçõesproblemas inspiradas na realidade do mundo do trabalho. A aprendizagem ocorre de forma colaborativa, integrada, interdisciplinar e transdisciplinar, utilizando pequenos grupos e operando no contexto clínico e social. 
Para possibilitar tal proposta de integração curricular, o projeto pedagógico do curso propõe uma integração do trabalho docente e discente, por meio de tecnologias digitais, a partir do conceito da aprendizagem híbrida, ou seja, a mesclagem de diferentes métodos de ensino-aprendizagem em modalidades presencial e online ${ }^{9,11}$. Essa iniciativa entra em sintonia com a flexibilização das propostas curriculares que vêm sendo estimulada pelas políticas públicas nacionais ${ }^{15}$. Recentemente, o Ministério da Educação aumentou de $20 \%$ para $40 \%$ o limite de carga horária ofertada para disciplinas, com metodologia a distância, em cursos de graduação presencial de Instituição de Educação Superior ${ }^{16}$.

Dessa forma, o AVA institucional, chamado AVA-UFPE, desenvolvido a partir do moodle, plataforma de software livre de apoio à aprendizagem mais difundida no mundo, foi customizado para atender as orientações do projeto pedagógico do curso $^{15}$. Para gestão dos processos, além da equipe técnica responsável pelo AVA, composta pelo Núcleo de Ensino a Distância que integra ações de toda a universidade, o Núcleo de Ciências da Vida possui em seu corpo docente uma professora em Saúde Digital, uma Coordenação docente do Ambiente Virtual de Aprendizagem (CAVA) e equipe técnica para suporte local.

O projeto pedagógico apresenta a ABP como um dos eixos centrais para o desenvolvimento curricular do curso. $\mathrm{Na}$
ABP, a ação pedagógica acontece em pequenos grupos de cerca de dez estudantes e um tutor, chamado de grupo tutorial. O tutor é um membro do corpo docente cuja função é dar suporte ao trabalho do grupo ao longo do processo tutorial, promover integração dos seus membros, potencializar o processo de aprendizagem dos estudantes e avaliar seus desempenhos por meio de avaliações formativas ${ }^{6}$. Dentre os alunos, um será o coordenador e outro será o relator, com revezamento entre as semanas tutoriais, para que todos tenham a oportunidade de exercer diferentes funções. Ao ser formado, o grupo estabelece um contrato de convivência, a fim de garantir o trabalho harmônico ao longo das sessões tutoriais ${ }^{13}$.

Os ciclos tutoriais duram uma semana e são desenvolvidos a partir de uma sequência de sete passos, divididos em três momentos: dois presenciais (abertura e fechamento da semana) e um outro assíncrono em Ambiente Virtual de Aprendizagem (ao longo da semana tutorial). Recursos de e-learning são mobilizados em todas as etapas para apoiar o processo da ABP, como os fóruns de discussão online, disponibilização de situações-problemas e guias do tutor sincronizados com o calendário de cada módulo e com controle de acesso, além da avaliação formativa do estudante pelo tutor com feedback semanal. Dessa forma, com a intensidade de uso de recursos online e a mesclagem de modalidades presencial e 
a distância, a aprendizagem baseada em problemas se integra ao blended learning, em uma ABP híbrida.

No primeiro encontro presencial, os passos um até o cinco da ABP são desenvolvidos. O primeiro se caracteriza pela leitura do problema, identificação e esclarecimento de termos desconhecidos. Os alunos e tutores leem a situação problema que é exibida em televisor conectado a um computador que está exibindo o AVA. A situação foi programada para estar disponível na plataforma, no momento do início da sessão tutorial. O grupo parte para a identificação de possíveis termos, conceitos e elementos pouco compreendidos ou desconhecidos. O conhecimento a priori dos estudantes pode ser usado para tentar elucidar seus significados rapidamente. Caso não seja possível, os termos devem ser levados à problematização. Essa etapa é importante para ampliar o vocabulário dos estudantes e fortalecer a compreensão de conceitos ${ }^{7}$.

No segundo passo, o grupo deve definir o problema central a ser trabalhado. Nesse processo, os estudantes podem dividi-lo em partes e identificar elementos relevantes da situação apresentada e, juntos, construírem uma síntese textual que deverá ser explicado nos passos seguintes. Além de exercitar a capacidade de síntese, os estudantes precisam buscar consensos para definir um problema de forma satisfatória a todos do grupo ${ }^{2,7}$.

A discussão segue para o terceiro passo, no qual os estudantes analisam o problema e tentam explicá-lo. A partir de conhecimentos previamente adquiridos, experiências de vida e vivências acadêmicas do estágio no serviço de saúde, o grupo deve tentar formular explicações de conceitos e processos envolvidos na situação problema7 ${ }^{7}$ Esse processo é importante, pois os estudantes estão mais aptos a construir um novo conhecimento quando este for fundamentado em, ou contextualizado com, algo que ele já saiba1. Essa etapa é denominada "brainstorming", uma vez que os estudantes são encorajados a verbalizar livremente suas opiniões sobre determinado assunto para que o grupo produza ideias adicionais a partir da incorporação ou reconstrução dos conhecimentos e das suposições expostas ${ }^{2}$.

No quarto passo, o grupo deve organizar as ideias que surgiram para tentar obter uma visão geral do problema, definir as hipóteses explicativas sobre sua natureza e identificar as lacunas de conhecimento. As hipóteses geradas pelo grupo devem ilustrar explicações com base em mecanismos, de forma a entender os conceitos, evitando explicações simplificadas e superficiais. A participação de todos na construção das hipóteses é essencial, pois cada um dos estudantes deve se identificar com o produto da discussão em grupo, a fim de encontrar o estímulo para o estudo individual ${ }^{13}$.

Após a formulação das hipóteses, dá-se início ao quinto passo que é 
dedicado à formulação dos objetivos de aprendizagem. Eles deverão responder às hipóteses formuladas e irão orientar 0 estudo do problema em questão ${ }^{2,7}$.

Ao término do primeiro encontro presencial, o registro dos termos desconhecidos (passo 1), do problema central (passo 2), a organização das ideias e das hipóteses que surgem no brainstorm (passo 3 e 4), assim como os objetivos de estudo (passo 5) são sistematizados e registrados em uma relatoria que deve ser disponibilizada no AVA, pelo relator, a todos membros do grupo, como um novo tópico em um fórum de discussão. Para isso, o estudante relator é incentivado a usar dispositivos móveis, como tablet ou notebook, para facilitar a coleta, síntese e publicação das informações. Esse documento será a memória do que foi discutido e servirá, tanto aos estudantes, quanto ao tutor, como orientação para o estudo e as discussões seguintes.

$\mathrm{Na}$ ABP tradicional, o sexto passo consiste no estudo individual. Os membros do grupo usam seus próprios meios e estratégias para coletar, individualmente, informações a respeito dos assuntos levantados nos objetivos de aprendizagem. Esse passo estimula o estudante a desenvolver habilidades para gerenciar o próprio aprendizado e buscar ativamente as informações. Ao longo do processo, o estudante pode identificar sua maneira de aprender de forma mais eficaz, tendo ajuda do tutor para facilitar essa identificação ${ }^{1,7}$. Esse é seguido do sétimo e último passo que consiste no compartilhamento dos estudos e pesquisas realizados pelos estudantes ${ }^{2}$.

No curso de medicina do NCV, os passos seis e sete descritos são mesclados e envolvem uma atividade a distância. À medida que os alunos avançam no estudo individual, eles são incentivados a compartilharem os seus achados e desenvolverem uma discussão assíncrona em um fórum digital. Nele, os participantes podem trocar opiniões e debater as hipóteses e objetivos de aprendizagem levantados. Nesse espaço, o estudante compartilha suas sínteses, reflexões e referências, acompanha o andamento das discussões e retira suas dúvidas, contribuindo com a construção do conhecimento coletivo.

O caráter de comunicação assíncrona, no qual uma pessoa posta uma mensagem e outra pessoa a lê e responde em um momento posterior, resulta em uma construção de linhas de discussão ao longo do tempo ${ }^{17}$. O fato de o estudante se engajar na discussão online, respeitando seu próprio ritmo, permite o desenvolvimento de respostas mais elaboradas, pois o estudante tem mais tempo para ler e pensar sobre elas. Nesse quesito, os tutores também são beneficiados pela possibilidade de um maior tempo para analisar o rumo da discussão e nela intervir de forma mais assertiva. De todo modo, a possibilidade de um maior tempo para refletir durante a elaboração de uma pergunta/resposta 
resulta em uma discussão mais profunda acerca do problema que a equipe está buscando explicar/solucionar ${ }^{17,18,19,20}$.

Discussões que acontecem virtualmente fornecem um registro eletrônico que pode ser acessado, repetidamente e a qualquer momento, pelos estudantes, servindo, muitas vezes, de material para revisão dos assuntos estudados. Além disso, a discussão a distância também pode encorajar estudantes que sentem mais dificuldades de participar de um debate presencial, oferecendo, assim, condições mais equilibradas de modo que todos os estudantes contribuam para o crescimento do grupo ${ }^{17,18}$.

Para fechar o ciclo tutorial, é realizado um novo encontro presencial para execução do sétimo passo que consiste na discussão do estudo individual e compartilhado no $\mathrm{AVA}^{7}$. O novo conhecimento é usado para explicar, de forma fundamentada, a situação problema. Cada estudante deve explicitar a síntese das suas pesquisas de forma contextualizada, aplicando os novos conhecimentos para resolução dos problemas levantados no primeiro encontro. Esse processo promove um aprendizado mais significativo e uma maior retenção do conhecimento ${ }^{1}$. Os componentes do grupo são estimulados a ouvir e entender as ideias trazidas pelos colegas, de forma a complementar suas sínteses individuais, por meio da socialização do conhecimento e da ajuda mútua. A colaboração provocada pela $A B P$ é essencial, tanto na perspectiva cognitiva, quanto na perspectiva motivacional sóciocomportamental ${ }^{1}$. Nesse encontro, o tutor deve estimular a análise crítica, tanto da fonte bibliográfica utilizada, como da própria informação compartilhada ${ }^{13}$. Além disso, os estudantes são estimulados a apresentar tópicos ou pontos de vista diferentes do que foi abordado no ambiente virtual, de forma a complementar e aprofundar a discussão.

Após a sessão tutorial, o relator disponibiliza a síntese das discussões no ambiente virtual. $O$ tutor avalia os estudantes, preenchendo critérios de avaliação específicos disponibilizados no AVA referentes ao seu desempenho, tanto nos encontros presenciais quanto no ambiente virtual. O registro das discussões no fórum digital, assim como as avaliações prévias, pode ser revisitado para auxiliar na avaliação da participação do estudante e a sua evolução ao longo do tempo.

$O$ registro das atividades possibilita uma melhoria na confiabilidade da avaliação na ABP, pois permite ao tutor avaliar o estudante em duas situações diferentes: pelo seu desempenho presencial e pelo virtual ${ }^{17}$. A partir da concepção da Avaliação Formativa ${ }^{21}$, o feedback (devolutiva) do tutor para cada estudante também é realizado e registrado. Para tanto, o AVA disponibiliza um recurso dedicado à avaliação, no qual a comunicação tutor-estudante é facilitada 
por meio de espaços para comentários abertos e trocas de mensagens.

Apesar das inúmeras vantagens na facilitação do processo ensinoaprendizagem utilizando a ABP híbrida entre os quais se destacam a sistematização e aprofundamento do conteúdo, o desenvolvimento da habilidade de síntese e uma maior organização do pensamento - algumas dificuldades foram identificadas, como o tempo necessário para elaboração de postagens com qualidade e 0 não entendimento, por parte de alguns estudantes, da importância da participação no fórum de discussão. Contudo, a experiência tem demonstrado que, nas semanas em que a discussão virtual não foi bem trabalhada, a qualidade da discussão no encontro presencial também foi prejudicada; e que, na medida em que os alunos avançam no curso, ocorre uma maior apropriação do AVA e são desenvolvidas novas habilidades que os ajudam a superar as dificuldades anteriormente $\operatorname{citadas}^{18}$. O que suscita a necessidade de estudos adicionais para compreender melhor os fatores envolvidos.

\section{Conclusão}

A aprendizagem baseada em problemas e o e-learning estão cada vez mais presentes nas escolas médicas da atualidade, enquanto possibilidade metodológica e realidade da integração das novas tecnologias educacionais. Assim, o ePBL se apresenta como um caminho para potencializar as práticas já consolidadas da aprendizagem baseada em problemas, como a ABP híbrida.

A partir da descrição e análise da experiência relatada neste artigo, pode-se perceber que a versão híbrida da $\mathrm{ABP}$ aumenta os campos de interação entre os membros do grupo tutorial, promove discussões mais profundas, estimula a aprendizagem colaborativa, apoia o estudante no estudo autodirigido e oferece condições mais equilibradas para que estudantes com diferentes personalidades se envolvam no processo tutorial. Além disso, a flexibilidade de tempo e espaço, ao realizar atividades a distância, amplia as possibilidades do ensinar e aprender.

Com este relato de experiência, procura-se apontar a integração das novas tecnologias educacionais com a ABP como um caminho para um potente desenvolvimento curricular das escolas médicas. Assim, recomenda-se a contemplação da aprendizagem híbrida nos projetos pedagógicos dos cursos de graduação, a presença de professores em saúde digital no corpo docente, a incorporação das atividades de avaliação do estudante no AVA e a criação de condições para desenvolvimento, manutenção e suporte de plataformas digitais. 


\section{Referências}

1. Bate E; Hommes J; Duvivier R; Taylor DC. Problem-based learning (PBL): Getting the most out of your studentsTheir roles and responsibilities: AMEE Guide. No. 84. 2014; 36(1): 1-12. [Acesso em: 15 de julho de 2019]. Disponível em: https://www.ncbi.nlm.nih.gov/pubmed/242 95273

2. Schmidt HG. Problem-based learning: Rationale and description. Medical education. 1983; 17(1): 11-16. [Acesso em: 15 de julho de 2019]. Disponível em: https://www.ncbi.nlm.nih.gov/pubmed/682 3214

3. Barrows HS. A taxonomy of problem-based learning methods. Medical education. 1986: 20(6): 481-486. [Acesso em: 15 de julho de 2019]. Disponível em: https://doi.org/10.1111/j.1365-

2923.1986.tb01386.x

4. Davis MH; Harden RM. AMEE Medical Education Guide N 15: Problembased learning: a practical guide. Medical teacher. 2009; 21(2): 130-140. [Acesso em: 18 de julho de 2019]. Disponível em: https://www.tandfonline.com/doi/abs/10.10 80/01421599979743

5. Harden RM; Davis MH. The continuum of problem-based learning. Medical teacher. 1998; 20(4): 317-322. [Acesso em: 17 de julho de 2019]. Disponível em:

https://www.tandfonline.com/doi/abs/10.10 80/01421599880733

6. Souza SCD; Dourado L. Aprendizagem baseada em problemas (ABP): um método de aprendizagem inovador para o ensino educativo. Holos. 2015; 5: 182-200. [Acesso em: 15 de julho de 2019]. Disponível em:

http://www2.ifrn.edu.br/ojs/index.php/HOL OS/article/view/2880

7. UFPEa. Campus Agreste. Núcleo de Ciências da Vida. Curso de Medicina. Manual do Tutor. Caruaru: UFPE; 2014. 8. Ellaway R; Masters K. AMEE Guide 32: e-Learning in medical education Part 1: Learning, teaching and assessment. Medical teacher. 2008; 30(5): 455-473. [Acesso em: 15 de julho de 2019]. Disponível em: https://www.ncbi.nlm.nih.gov/pubmed/185 76185

9. Bacich L; Tanzi Neto A; Trevisani FM. Ensino híbrido: personalização e tecnologia na educação. Porto Alegre: Penso Editora; 2015.

10. Magnagnagno CC; Ramos MP; Oliveira LMPD. Estudo sobre o uso do moodle em cursos de especialização a distância da UNIFESP. Rev. bras. educ. méd. 2015; 39(4): 507-516. [Acesso em: 15 de julho de 2019]. Disponível em: http://www.scielo.br/scielo.php?pid=S0100

55022015000400507\&script=sci_abstract \&tlng $=\mathrm{pt}$

11. Magalhães GSG; Portella NM; Padilha MA. Aprendizagem híbrida e integração curricular: potencialidades da coreografia institucional em uma nova escola médica no brasil. In: Vaquero TE; Brescó BE; Coiduras RJL; Carrera X. EDUcación con TECnología: un compromiso social. Iniciativas y resultados de investigaciones y experiencias de innovación educativa. Lleida: Edicions de la Universitat de Lleida; 2018. [Acesso em 25 de julho de 2019]. Disponível em: https://doi.org/10.21001/edutec.2019. 12. Escolas Médicas do Brasil. Todas as Escolas Médicas. [Acesso em 27 de julho de 2019]. Disponível em: http://www.escolasmedicas.com.br. 13. Borges MC; Chachá SGF; Quintana SM; Freitas LCC; Rodrigues MDLV. Aprendizado baseado em problemas. Medicina (Ribeirão Preto. Online). 2014; 47(3): 301-307. [Acesso em: 18 de julho de 2019]. Disponível em: http://www.revistas.usp.br/rmrp/article/vie w/86619

14. Brasil. Ministério da Educação. Resolução no 3, de 20 de junho de 2014. Institui Diretrizes Curriculares Nacionais do Curso de Graduação em Medicina e dá outras providências. [internet]. Diário Oficial [da] República Federativa do Brasil, 2014 jun. 23; 1: 8-11. [Acesso em: 27 de julho de 2019]. Disponível em: http://portal.mec.gov.br/index.php?option= com_docman\&view=download\&alias $=158$ 74-rces003-14\&category_slug=junho2014-pdf\&Itemid=30192 
15. UFPEb. Campus Agreste. Núcleo de Ciências da Vida. Curso de Medicina. Projeto Pedagógico do Curso de Medicina. Caruaru: UFPE; 2014.

16. Brasil. Ministério da Educação. Portaria no 1.428 , de 28 de dezembro de 2018. Dispõe sobre a oferta, por Instituições de Educação Superior - IES, de disciplinas na modalidade a distância em cursos de graduação presencial.[internet]. Diário Oficial [da] República Federativa do Brasil, 2018 dez. 31; 1:250. [Acesso em: 27 de julho de 2019]. Disponível em:

http://www.in.gov.br/materia//asset_publisher/Kujrw0TZC2Mb/content/i d/57496468/do1-2018-12-31-portaria-n-1428-de-28-de-dezembro-de-201857496251

17. Alamro AS; Schofield S. Supporting traditional PBL with online discussion forums: A study from Qassim Medical School. Medical teacher. 2012; 34(sup1): S20-S24. [Acesso em: 8 de agosto de 2019]. Disponível em: https://www.ncbi.nlm.nih.gov/pubmed/224 09186

18. Crawford TR. Using problembased learning in web-based components of nurse education. Nurse education in practice. 2011; 11(2): 124-130. [Acesso em: 8 de agosto de 2019]. Disponível em: https://www.ncbi.nlm.nih.gov/pubmed/211 11680

19. Donlan P. Use of the Online Discussion Board in Health Professions Education: Contributions, Challenges, and Considerations. Journal of Continuing Education in the Health Professions. 2019; 39(2): 124-129. [Acesso em: 8 de agosto de 2019]. Disponível em: https://europepmc.org/article/med/309985 68

20. Silva ASR; Oliveira LR; Cavalcante LE; Rolim RM; Sousa LF. Metodologias ativas de ensino e aprendizagem em educação à distância em saúde. Revista de Saúde Digital e Tecnologias

Educacionais.2018; 3(1): 15-26. [Acesso em: 14 de outubro de 2019]. Disponível em:

http://periodicos.ufc.br/resdite/article/view/ 40059

21. Dent J; Harden RM; Hunt D. A practical guide for medical teachers.
Amsterdam: Elsevier health sciences, 2017. 


\section{Como citar este artigo}

Portela, NM.; Costa, J. MBS.; Magalhães, GSG. A experiência com o uso do e-learning na aprendizagem baseada em problemas de um curso de medicina. Revista de Saúde Digital e Tecnologias Educacionais. [online], volume 5, n. 1. Editor responsável: Luiz Roberto de Oliveira. Fortaleza, mês e ano, p. 01-12. Disponível em: http://periodicos.ufc.br/resdite/index. Acesso em "dia/mês/ano".

Data de recebimento do artigo: 15/10/2019

Data de aprovação do artigo: 11/12/2019

Data de publicação: 17/04/2020 